
zons farence
EGM:
CENTRO DE ESTUDIOS
INTERDISCIPLINARIOS SOBRE MUJERES
MU MAESTRIA "PODER Y SOCIEDAD

\title{
La traducción de textos queer y feministas: visibilizar a través de la lengua
}

\author{
Luciana M. Capurro* \\ Paula A. Dechima* * \\ Gabriela Saizar** * \\ Karen Vera****
}

\section{Resumen}

La presente reflexión tiene como objetivo analizar los problemas de traducción del inglés al español relacionados con los asuntos de género en el ámbito del texto académico y comentar las decisiones que tomamos para resolverlos. Los textos analizados y traducidos reflexionan sobre la (in)visibilización de las mujeres y personas del colectivo LGBTIQ+y el trato que reciben como grupos minorizados en textos académicos y su traducción. Tradujimos textos de cuatro autores que

* Traductora técnico-científica inglés-español. IES en Lenguas Vivas “J. R. Fernández". Contacto: lucianamcapurro@gmail.com

* * Traductora técnico-científica inglés-español. IES en Lenguas Vivas “J. R. Fernández". Contacto: paula.dechima@gmail.com

* * * Traductora técnico-científica inglés-español. IES en Lenguas Vivas “J. R. Fernández". Contacto: saizargabriela@gmail.com

* * * * Traductora técnico-científica inglés-español. IES en Lenguas Vivas "J. R. Fernández". Contacto: karendanielavera@gmail.com

Capurro, Luciana; Dechima, Paula; Saizar, Gabriela; Vera, Karen. "La traducción de textos queer y feministas: visibilizar a través de la lengua" en Zona Franca. Revista del Centro de estudios Interdisciplinario sobre las Mujeres, y de la Maestría poder y sociedad desde la problemática de Género, N²9, 2021 pp. 272-287. ISSN, 2545-6504 Recibido: 30 de julio 2021; Aceptado: 19 de octubre 2021.

Revista Zona Franca- Centro de estudios interdisciplinario sobre las mujeres (CEIM)- Maestría poder y sociedad desde la problemática de género (MG), Rosario, Argentina. ISSN, 2545-6504 http://zonafranca.unr.edu.ar/index.php/ZonaFrancal Número 29 (2021). 
abordan estos temas desde distintas perspectivas en distintos momentos históricos. En estos textos identificamos problemas de traducción, a partir de los cuales nos cuestionamos nuestro rol como traductoras y reflexionamos acerca de las intervenciones que nos podíamos permitir en el proceso de traducción. Este trabajo se estructura a partir de tres problemas principales: neologismos, juegos de palabras y lenguaje no binario.

Palabras clave: problemas de traducción, género en la traducciónneologismos, juegos de palabras, lenguaje no binario

\section{Translating queer and feminist texts: giving visibility through language}

\section{Abstract}

The following article presents the analysis of translation problems from English into Spanish, regarding issues of gender in academic texts, and shows the decisionmaking process we underwent to solve them. The texts analysed and translated shed light on the (in)visibilization of women and members of the LGBTIQ+ community and the treatment they receive as minorised groups in academic texts and their translation. We translated texts from four authors who discuss these issues from different perspectives in different historical moments. In these texts, we identified translation problems, which made us question our role as translators and reflect upon the extent to which we could intervene in the translation process. The structure of this article is based on three main translation problems: neologisms, wordplay and non-binary language.

Keywords: translation problems, gender in translation, neologisms, worldplay, non-binary language

\section{Introducción}

Este trabajo se realizó en el marco de la Residencia del Traductorado TécnicoCientífico en Inglés, la cual tuvo lugar de septiembre a diciembre del 2020 y es la asignatura final de la carrera en el IES en Lenguas Vivas “J. R. Fernández". Los textos fueron solicitados por la profesora Verónica Storni Fricke para el Proyecto de investigación "Nuevos enfoques en la teoría, crítica y didáctica de la traducción:

Revista Zona Franca- Centro de estudios interdisciplinario sobre las mujeres (CEIM)- Maestría poder y sociedad desde la problemática de género (MG), Rosario, Argentina. ISSN, 2545-6504 http://zonafranca.unr.edu.ar/index.php/ZonaFranca| Número 29 (2021). 
traducción poscolonial, feminista y Queer", desarrollado en la misma institución. En la redacción de este artículo, evitamos generalizar mediante el género masculino, por lo que optamos por el lenguaje no binario directo, es decir, el uso del morfema $\{-\mathrm{e}\}$.

Al finalizar esta asignatura, reflexionamos sobre los problemas de traducción del inglés al español relacionados con los asuntos de género en el ámbito del texto académico y comentamos las decisiones que tomamos para resolverlos. Según Christiane Nord, "un problema de traducción es un problema objetivo que todo traductor (...) debe resolver en el transcurso de una tarea de traducción determinada" (en Hurtado Albir, 1988a/1991:151). Los tres problemas en los que decidimos ahondar fueron los neologismos, los juegos de palabras y el lenguaje no binario, a partir de la traducción de textos de cuatro autores que abordan la (in)visibilización de las mujeres y personas del colectivo LGBTIQ+ y el trato que reciben como grupos minorizados tanto en textos académicos como en su traducción.

Los primeros tres textos analizados pertenecen al libro Queering TranslationTranslating the Queer. Theory, Practice, Activism [Queerizar la traducción y traducir lo queer. Teoría, práctica, activismo], editado por los autores Brian James Baer y Klaus Kaindl. Esta revolucionaria publicación expone investigaciones críticas acerca de los aspectos queer dentro del campo de los estudios de traducción.

El primer texto con el que trabajamos fue "Beyond Either/Or" [Más allá de uno $u$ otro], en el cual el autor Brian James Baer pone de relieve la importancia de la traducción y la diversidad de lenguas para comprender mejor las vivencias, experiencias y luchas de personas gays o queer de distintas partes del mundo. En este sentido, plantea diferentes realidades y formas de vivir la sexualidad ajenas a la perspectiva occidental. En particular, se discute el significado y la connotación negativa que tienen las palabras relacionadas con la homosexualidad que provienen

Revista Zona Franca- Centro de estudios interdisciplinario sobre las mujeres (CEIM)- Maestría poder y sociedad desde la problemática de género (MG), Rosario, Argentina. ISSN, 2545-6504 http://zonafranca.unr.edu.ar/index.php/ZonaFranca| Número 29 (2021). 
de los Estados Unidos en países como Rusia, en contraste con las creencias actuales de Occidente.

Debido a la globalización, la influencia del mundo occidental comenzó a exportar su visión del mundo, de manera tal que se estableció que esa sexualidad y cultura representaban a todas las personas que se identificaban como homosexuales. Sin embargo, debido a la escasa literatura producida en lenguas distintas al inglés, las culturas consideradas "periféricas" se ven obligadas a recurrir a textos traducidos para informarse y formar parte de la discusión. Por este motivo, Baer señala que el ámbito académico debería prestarle más atención a la traducción para así tener acceso a una pluralidad de voces y, en consecuencia, a un conocimiento más profundo de la sexualidad en distintas culturas. Asimismo, se podrá prevenir la homogeneización de las culturas a través de la única categorización de las vivencias de sus integrantes según la lengua hegemónica.

El siguiente texto fue "The Future Is a Foreign Country. Translation and Temporal Critique in the Italian It Gets Better Project" [El futuro es un país extranjero. Traducción y crítica temporal del proyecto italiano It Gets Better], en el cual su autora, Serena Bassi, explora la manera en la que está construido el discurso occidental en torno a la subjetividad sexual a través del análisis de la versión italiana del proyecto It Gets Better. Esta iniciativa nacida en Estados Unidos busca difundir un mensaje esperanzador y positivo por medio de videos publicados en YouTube, en los que se muestran las historias personales de integrantes del colectivo LGBTIQ+. Todas estas historias se enfocan en un pasado difícil que les protagonistas tuvieron que superar para dar lugar a un presente pleno y feliz, en el cual logran formar una familia y cumplir sus sueños. Sin embargo, la propuesta no fue imitada en otros países, por lo que la autora se basa en el ejemplo italiano y analiza los testimonios de cuatro personas para cuestionar los conceptos hegemónicos de "tiempo" y "cambio" propuestos por les creadores del proyecto.

Revista Zona Franca- Centro de estudios interdisciplinario sobre las mujeres (CEIM)- Maestría poder y sociedad desde la problemática de género (MG), Rosario, Argentina. ISSN, 2545-6504 http://zonafranca.unr.edu.ar/index.php/ZonaFranca| Número 29 (2021). 
Además, Bassi propone ampliar la noción de localización, entendida como una modalidad de traducción, para incluir fenómenos que no forman parte estrictamente de esa disciplina, como el proyecto It Gets Better. Este proyecto pasa por un proceso de localización completa, es decir que se transforma para crear un documento meta totalmente nuevo, lo cual le permite a la autora queerizar la traducción, ya que debe alejarse de las típicas estructuras binarias como el par texto fuente (de origen) y texto meta (de destino). El objetivo principal de la autora es brindar una base para permitir el desarrollo de una epistemología queer que perciba la sexualidad como un discurso moderno en el cual se incluyan distintos aspectos como el género, la nacionalidad y la etnia.

El último texto que analizamos y tradujimos de esta publicación fue "Queering Lexicography: Balancing Power Relations in Dictionaries" [Queerizar la lexicografía. Equilibrar las relaciones de poder en los diccionarios], en el cual la autora Eva Nossem analiza el proceso de producción lexicográfica desde una perspectiva queer, dos visiones que en principio parecen contradictorias, pero que la autora demuestra que se pueden conciliar para entender mejor las relaciones de poder que se establecen en estos procesos. Nossem describe el rol del diccionario en la cultura como esa figura de autoridad que busca mantener la apariencia de objetividad para generar confianza en sus usuaries y transmitir conocimientos. Sin embargo, en el análisis del proceso de producción de diccionarios monolingües, así como de los multilingües, se empieza a quebrar esta ilusión de objetividad y se pueden observar ciertas decisiones claramente subjetivas.

En ese sentido, la primera decisión que se toma ocurre en el proceso de armado de un corpus inicial, ya que, al valerse de obras anteriores, se corre el peligro de reproducir y fijar valores y normas que no dan lugar al desarrollo de nuevas perspectivas. La segunda decisión tiene que ver con los lemas que aparecerán en el diccionario, es decir, a qué palabras se les dará visibilidad y a qué otras se dejará de lado. Además, dado que no se pueden incluir todas las formas de cada palabra, se define una de esas formas como "neutral" para aparecer en la

Revista Zona Franca- Centro de estudios interdisciplinario sobre las mujeres (CEIM)- Maestría poder y sociedad desde la problemática de género (MG), Rosario, Argentina. ISSN, 2545-6504 http://zonafranca.unr.edu.ar/index.php/ZonaFranca| Número 29 (2021). 
entrada de diccionario. En el caso de los sustantivos, esta forma es el masculino singular, lo cual apoya la reproducción del discurso hegemónico que privilegia las formas y perspectivas masculinas sobre las demás. Por último, se decide cuál será la definición de cada palabra que, lejos de ser objetiva y seguir una guía estándar, evidencia el poder de le lexicógrafe, quien elige la información que considera pertinente incluir. Incluso puede decidir marcar las palabras aún más mediante etiquetas como "común" o "vulgar", las cuales sesgan la percepción de le usuarie al leerlas.

Por último, trabajamos con los dos primeros capítulos del libro Translation and Gender. Translating in the 'Era of Feminism' [Traducción y género. Traducir en la "era del feminismo"], escrito por Luise von Flotow. En el primer capítulo, "Historical Background" [Contexto histórico], resume cómo los movimientos de mujeres problematizaron la lengua a partir de 1960. En primer lugar, la autora se enfoca en el surgimiento de la idea de género como construcción social. Sobre la base de un aforismo de Simone de Beauvoir, las feministas angloestadounidenses comenzaron a utilizar el término 'género' para referirse al proceso social mediante el cual una niña se convierte en mujer según los atributos típicos de una determinada época y un lugar en particular. Ese fue el inicio de una etapa de revisión y (re)descubrimiento del material académico, literario y científico producido por mujeres.

En segundo lugar, von Flotow se aboca a la manera en la que las mujeres empezaron a cuestionar la lengua desde distintas perspectivas. Según la visión reformista, la lengua es un síntoma de la sociedad que la vio nacer y es posible modificarla. Para conseguirlo, se crearon manuales, se dictaron talleres y se fundaron organizaciones estatales para representar mejor a la mujer en la lengua. Por otro lado, la visión radical considera que la sintaxis y los géneros literarios perpetúan las estructuras de poder patriarcales y que es necesario reconstruirlos porque representan un peligro para las mujeres.

Revista Zona Franca- Centro de estudios interdisciplinario sobre las mujeres (CEIM)- Maestría poder y sociedad desde la problemática de género (MG), Rosario, Argentina. ISSN, 2545-6504 http://zonafranca.unr.edu.ar/index.php/ZonaFranca| Número 29 (2021). 
Finalmente, la autora hace una breve mención a la traducción en relación con el género y comenta que las mujeres han tenido que traducirse cuando aparecen en la esfera pública, ya que el lenguaje fue creado para describir y representar las experiencias de los hombres y limitar las posibilidades de las mujeres. En este sentido, para producir nueva literatura, deben desenterrar esos textos y conocimientos oprimidos y expresarlos en un lenguaje que las excluye. Esta sección es una introducción al resto de la obra, en la que se analiza en profundidad muchas de las complejidades que surgen cuando se estudia el género junto con la traducción.

En el segundo capítulo, "Gender and the Practice of Translation" [El género y la práctica de la traducción], von Flotow describe la relación entre el género y la traducción en el marco de una "era del feminismo", que puso a prueba las convenciones tradicionales sobre los tipos de textos que se traducían y cómo se traducían. La década de los setenta vio nacer una oleada de textos feministas radicales y experimentales que buscaban socavar las estructuras típicas del patriarcado a través de usos innovadores de la lengua. La traducción fue la forma de llevar estas herramientas a otras culturas y, al mismo tiempo, la forma de descubrir alternativas y vivencias más allá de Europa occidental y Norteamérica. Quienes traducían tenían que plantearse cómo llevar estos cambios gramaticales, léxicos, culturales o semánticos de una lengua a otra para que funcionen con el efecto deseado, a pesar de contar con opciones completamente distintas.

Ante esas complejidades, les traductores tuvieron que decidir qué actitud tomar, no solo porque era necesario explicar en muchos casos el proceso de traducción y los cambios que se habían hecho cuando la lengua meta no lograba transmitir el mismo significado que la lengua fuente, sino también porque algunes traductores, sobre todo traductoras feministas, decidían "corregir" textos que tenían una gran carga machista para alinearlos con esta nueva era que luchaba por la igualdad entre las mujeres y los hombres. Tanto en las traducciones comentadas como en las traducciones intervencionistas, le traductore pasaba a un plano de

Revista Zona Franca- Centro de estudios interdisciplinario sobre las mujeres (CEIM)- Maestría poder y sociedad desde la problemática de género (MG), Rosario, Argentina. ISSN, 2545-6504 http://zonafranca.unr.edu.ar/index.php/ZonaFranca| Número 29 (2021). 
mayor protagonismo, un hecho que de por sí subvierte las normas implícitas de "invisibilidad" de la traducción. La conciencia de género y la traducción en la era del feminismo dieron lugar a una reescritura de los cánones y a un descubrimiento de alternativas a las de la sociedad patriarcal.

\section{Neologismos: darle entidad a lo invisibilizado}

El Diccionario de la Real Academia Española (DRAE) define al neologismo como "vocablo, acepción o giro nuevo en una lengua", es decir, es el acto de crear nuevas palabras. Los neologismos suponen un problema de traducción lingüístico, que está relacionado con "el código lingüístico, fundamentalmente en el plano léxico y morfosintáctico (...). Derivan en gran parte de las diferencias entre lenguas. Pueden ser de comprensión y/o de reexpresión" (Hurtado Albir, 2011:288).

Los neologismos fueron un recurso abundante en todos los textos para nombrar distintas realidades y experiencias y darle entidad a lo invisibilizado. Por un lado, en el texto de Bassi, aparecen los extranjerismos queer y gay, por lo que consultamos a personas fuera del ámbito de la traducción para corroborar su frecuencia de uso en el lenguaje cotidiano y así decidir si entenderlo como un préstamo naturalizado o como un término extranjero.

En el caso de la palabra 'gay', notamos que está ampliamente aceptada en el español rioplatense, de manera que mantuvimos la grafía original, pero respetamos las reglas gramaticales de la lengua española, es decir, la escribimos en redonda y en plural cuando fue necesario, como en el siguiente ejemplo: "a better future awaits lesbian, gay, bisexual, and transgender people, who should therefore remain hopeful and push past the pain" [les espera un mejor futuro a las personas lesbianas, gays, bisexuales y transgénero, quienes deberían, por lo tanto, albergar la esperanza y sobreponerse al dolor] (Bassi, 2017:58).

El caso de 'queer' no fue tan sencillo, ya que, al no ser de uso tan frecuente, consideramos que es un extranjerismo. Por lo tanto, decidimos no pluralizarla y

Revista Zona Franca- Centro de estudios interdisciplinario sobre las mujeres (CEIM)- Maestría poder y sociedad desde la problemática de género (MG), Rosario, Argentina. ISSN, 2545-6504 http://zonafranca.unr.edu.ar/index.php/ZonaFranca| Número 29 (2021). 
escribirla en cursiva. Sin embargo, como se trata de un término específico del área de Traducción y género, solo la primera mención en cada texto está marcada en cursiva para no afectar el ritmo de la lectura.

Por otro lado, en Translation and Gender, Luise von Flotow emplea los neologismos malestream y malespeak. Como estos términos no tienen un equivalente en español, los mantuvimos en inglés y, para que se comprenda el sentido, agregamos una nota al pie explicando el significado de cada uno. De esta manera, explicamos que malestream es un neologismo en inglés que surgió como crítica al sesgo masculino de la sociología. Describe una perspectiva masculina que asume que sus conclusiones pueden aplicar a toda la población, independientemente de su género o sexo biológico. Por su parte, malespeak es un eufemismo que se utiliza para hacer referencia a la violencia que ejercen los hombres sobre las mujeres, pero sin nombrarla. Por ejemplo, cuando se habla de "violencia de género" o "violencia familiar", lo más usual es que sea el hombre quien la ejerza, pero no se los menciona directamente.

Otro neologismo que utiliza la autora es la palabra gendered, que entendimos de maneras diferentes según el contexto en el que aparece. En el caso de "a rewriting of those gendered qualities and attitudes ascribed to women of other eras" (von Flotow, 1997:34), tradujimos gendered como "de género": "es una reescritura de esas cualidades y actitudes de género atribuidas a las mujeres de otras épocas". No obstante, en el caso de "the 'translator-effect', the mark each translator, as a gendered individual, leaves on the work" (von Flotow, 1997:35), tradujimos este término como "generizado": "el 'efecto traductor', la marca que cada traductora, como un individuo generizado, deja en la obra".

Por último, von Flotow también acude a un recurso ortográfico para crear neologismos. En su traducción del siguiente fragmento en francés, "Si nous continuons à nous parler le même langage, nous allons reproduire la même histoire. Recommencer les mêmes histoires" (Irigaray en von Flotow, 1977:205), la autora

Revista Zona Franca- Centro de estudios interdisciplinario sobre las mujeres (CEIM)- Maestría poder y sociedad desde la problemática de género (MG), Rosario, Argentina. ISSN, 2545-6504 http://zonafranca.unr.edu.ar/index.php/ZonaFranca| Número 29 (2021). 
busca mantener la ambigüedad de la palabra histoire [historia], que puede referirse tanto a la historia mundial como a cada historia individual. Por esta razón, la autora crea el neologismo (his)story. Asimismo, resalta entre paréntesis la presencia del adjetivo posesivo masculino his [de él], para poner de relieve que la historia está escrita por los hombres y describe sus experiencias: "If we continue to speak the same language, we will reproduce the same (his)story. Repeat the same (his)stories. (my translation)" (von Flotow, 1997:10) [Si continuamos hablando el mismo lenguaje, vamos a reproducir la misma historia. A repetir las mismas historias]. Esta coincidencia entre la raíz de la palabra y el adjetivo posesivo que sucede en el inglés no se replica en español, por lo que decidimos no reproducir el neologismo en nuestra traducción, sino mantener el sentido de la palabra y agregar una nota al pie explicando el neologismo encontrado en el texto fuente.

Por su parte, Eva Nossem, autora de "Queer(ing) Lexicography", no solo utiliza el neologismo queering, que refiere a la palabra 'queer' utilizada como adjetivo, sino que, además, mediante el uso de paréntesis, pone de relieve el proceso de queerizar la lexicografía. A diferencia de "(his)tory", en este caso logramos crear una versión en español que mantiene el mismo sentido e impacto y que se lee con la misma naturalidad:

Como se observó en Nossem (2015:110), “Queer(ing) Lexicography” [La lexicografía queer(izada)] suena como un oxímoron, ya que ambos conceptos parecen ser mutuamente excluyentes.

\section{Juegos de palabras: transformar para cuestionar}

Según Delabastita:

"Juego de palabras es el nombre genérico que se da a los numerosos fenómenos textuales en los que se aprovechan los rasgos estructurales de las lenguas para producir una confrontación que sea significativa en términos comunicativos de dos (o más) estructuras lingüísticas con formas

Revista Zona Franca- Centro de estudios interdisciplinario sobre las mujeres (CEIM)- Maestría poder y sociedad desde la problemática de género (MG), Rosario, Argentina. ISSN, 2545-6504 http://zonafranca.unr.edu.ar/index.php/ZonaFranca| Número 29 (2021). 
relativamente similares y significados relativamente diferentes". (1996:128, traducción nuestra)

Los juegos de palabras no solo representan un problema de traducción lingüístico, sino que además suponen un problema de traducción cultural, ya que las diferencias entre culturas podrían impedir la transmisión fiel de las referencias a las que aluden esos juegos de palabras. Sin embargo, como en este caso las lenguas de trabajo pertenecen a culturas occidentales relativamente similares, las referencias se pudieron conservar en la traducción al español. Por esta razón, nos centramos en la resolución del problema lingüístico.

En particular, Luise von Flotow utiliza los juegos de palabras a lo largo de toda su obra y dedica una sección del segundo capítulo a analizar específicamente la traducción de este recurso a partir del libro Gyn/Ecology (1978) de Mary Daly: "Mary Daly's book (...) is full of wordplay on aspects of American culture; she invents neologisms such as 'the-rapist', 'boreocracy' and the 'Totaled woman' to refer to more or less familiar ideas and then to undermine them with humour, irony, and anger" (von Flotow, 1997:21) [El libro (...) de Mary Daly está repleto de juegos de palabras sobre los aspectos de la cultura estadounidense; la autora inventa neologismos como 'violapeuta', 'aburocracia' y 'mujer Total' para referirse a ideas bastante conocidas y deslegitimarlas con humor, ironía y enojo].

En ese fragmento, von Flotow expone los neologismos que se inventaron a través de los juegos de palabras y explica la dificultad de traducirlos del inglés al alemán. Para traducirlos, en primer lugar, debatimos distintas opciones para resolver cada problema. En segundo lugar, clasificamos los juegos de palabras de Daly para decidir qué técnica íbamos a utilizar para traducir cada uno. Finalmente, seleccionamos las mejores según la combinación de palabras más adecuada y la naturalidad fonológica en español.

Revista Zona Franca- Centro de estudios interdisciplinario sobre las mujeres (CEIM)- Maestría poder y sociedad desde la problemática de género (MG), Rosario, Argentina. ISSN, 2545-6504 http://zonafranca.unr.edu.ar/index.php/ZonaFranca| Número 29 (2021). 
El primer tipo de juego de palabras utiliza la división de una palabra para crear una unidad de sentido conformada por dos palabras, como the-rapist. El segundo combina distintas palabras para remitir a ambas ideas y así crear un concepto nuevo, como bore-ocracy. Con el fin de mantener un paralelismo entre las estructuras de ambos tipos de juegos de palabras, decidimos utilizar la misma técnica, la combinación de dos palabras, y concluimos que la mejor alternativa era ampliarlos y explicar las palabras que se combinaban: "terapeuta" y "violador", algo como "violapeuta", para el primer ejemplo; "aburrimiento" y "burocracia", algo como "aburocracia", para el segundo. En el caso de Totaled woman [mujer Total], el tercer tipo, no fue necesario utilizar esta técnica dado que, en la traducción, pudimos combinar las dos palabras como en la lengua fuente sin necesidad de explicar el término.

Otro recurso analizado por la autora fue la homofonía, es decir, el uso de dos o más palabras que comparten el mismo sonido, independientemente de su grafía. La homofonía es un recurso lingüístico utilizado en la cultura anglófona muy difícil de recrear en la traducción al español. Esta dificultad radica en mantener el significado de las palabras utilizadas y encontrar homófonos equivalentes, que no abundan en nuestra lengua. Un claro ejemplo de homofonía, que fue imposible de replicar, se encuentra en la primera oración de esta sección, en la que se juega con la pronunciación de "Puns are punishment" (Levine en von Flotow, 1991:13). En ese caso, decidimos priorizar el sentido del mensaje, aunque perdiéramos la musicalidad del juego de palabras: "Los juegos de palabras están vinculados con el dolor; "son un castigo" (puns are punishment), escribió Suzanne Jill Levine (1991:13)".

\section{Lenguaje no binario: queerizar el discurso académico}

El tema que atravesaba todos los textos era el género y la traducción, por lo que analizamos los esfuerzos que hicieron les diferentes autores para no caer en el masculino genérico o para ofrecer una variante menos binaria. Como las posturas

Revista Zona Franca- Centro de estudios interdisciplinario sobre las mujeres (CEIM)- Maestría poder y sociedad desde la problemática de género (MG), Rosario, Argentina. ISSN, 2545-6504 http://zonafranca.unr.edu.ar/index.php/ZonaFranca| Número 29 (2021). 
de les autores fueron distintas, nuestras traducciones reflejan esa disparidad. En los textos escritos por Baer y por Bassi, por ejemplo, no observamos indicios de que les autores hubieran querido neutralizar la lengua. En nuestra traducción, de todas formas, decidimos evitar, siempre que fuera posible, la marca de género masculino de las palabras que, por la naturaleza del inglés, no marcan el género.

Al traducir el texto de Eva Nossem tomamos una perspectiva más radical, porque justamente la autora apuntaba contra el uso del masculino genérico en los diccionarios por parte de "les lexicógrafes", a quienes ella les adjudica una buena dosis de poder para moldear y definir la lengua. Nossem utiliza en inglés la tercera persona del plural para llamar la atención sobre la necesidad de salir del binarismo y habla de "the lexicographer themselves" (Nossem, 2017:174). Por ese motivo, en la traducción utilizamos el lenguaje no binario directo, para lo que adoptamos el uso del morfema $\{-e\}$ en lugar del morfema $\{-x\}$ con el fin de facilitar la lectura oral del texto.

Por último, Luise von Flotow se centra en resaltar el lugar de las mujeres en la historia, la literatura y la lengua, y ese interés se vuelca en su manera de escribir. Si bien no encontramos esfuerzos de la autora por salir del binarismo, sí está clara su postura de evitar el masculino genérico y utilizar el femenino con más frecuencia para no invisibilizar a las mujeres mediante la lengua. Von Flotow analiza en detalle el feminismo y la importancia de las mujeres en el mundo, pero su trabajo no abarca a las disidencias que existen más allá del modelo heterobinario. Enfocamos nuestro trabajo en las sutilezas: en qué casos se refería a un todo general y en cuáles se trataba realmente solo de mujeres.

Dado que el inglés por lo general no marca el género en los sustantivos, nos tuvimos que valer del contexto de publicación del texto fuente, de las sutilezas del estilo de la autora y, también, de nuestra propia subjetividad como traductoras para definir cuándo usar la forma femenina y cuándo era conveniente dejar el masculino generalizador. Podemos ver un ejemplo en el siguiente fragmento, en el que al

Revista Zona Franca- Centro de estudios interdisciplinario sobre las mujeres (CEIM)- Maestría poder y sociedad desde la problemática de género (MG), Rosario, Argentina. ISSN, 2545-6504 http://zonafranca.unr.edu.ar/index.php/ZonaFranca| Número 29 (2021). 
principio hablamos de "las traductoras" feministas de los años sesenta y setenta y, al final, de "los traductores" en general:

Translators are introducing and commenting on their work, and offering explanations for it. They are exerting further influence by writing scholarly essays and 'workshop reports' that draw attention to the work of translators and the historical, literary and biographical research that often accompanies a translated text. (von Flotow, 1997:35, itálicas nuestras)

[ Las traductoras están sumando elementos y haciendo comentarios acerca de su actividad, y ofrecen explicaciones sobre lo que hacen. Están ejerciendo una influencia mayor al escribir ensayos académicos e "informes de talleres" que dirigen la atención hacia el trabajo de los traductores y la investigación histórica, literaria y biográfica que muchas veces acompaña un texto traducido.]

Traducir a von Flotow hacia el final de la Residencia fue ilustrativo porque dedica parte de su obra a tratar el tema de la figura de les traductores. La autora comenta las distintas iniciativas que generaron las traductoras feministas, precursoras de una corriente que tiene como objetivo visibilizar no solo la labor de la traducción, sino también la propia subjetividad e ideología de las personas detrás de ese proceso.

\section{Conclusión}

La temática de los textos tratados pone constantemente sobre la mesa la idea de la visibilización de las minorías o grupos minorizados (tanto mujeres como personas del colectivo LGBTIQ+) por medio de la traducción. En ese sentido, entendemos la responsabilidad de nuestro rol activo como traductoras para transmitir de manera acertada la intención y los matices de los textos fuente, sin olvidar nuestra propia subjetividad ni el contexto de publicación de los textos meta. De la importancia de visibilizar esos grupos también surgen los ejes que

Revista Zona Franca- Centro de estudios interdisciplinario sobre las mujeres (CEIM)- Maestría poder y sociedad desde la problemática de género (MG), Rosario, Argentina. ISSN, 2545-6504 http://zonafranca.unr.edu.ar/index.php/ZonaFrancal Número 29 (2021). 
desarrollamos en este trabajo: los neologismos, los juegos de palabras y el uso del lenguaje no binario. Son tres recursos que ilustran algunos de los problemas de traducción que tuvimos que resolver al lidiar con textos que abordan la temática de género. En el proceso de traducción, tomamos conciencia de que ejercemos un intercambio cultural y de conocimiento, y de que no somos solamente traductoras, sino también escritoras y comunicadoras.

\section{Bibliografía}

BAER, Brian James (2017). "Beyond Either/Or. Confronting the Fact of Translation in Global Sexuality Studies". En Brian James Baer y Klaus Kaindl (eds.). Queering Translation-Translating the Queer. Theory, Practice, Activism. Reino Unido: Routledge, pp. 38-57.

BASSI, Serena (2017). "The Future Is a Foreign Country. Translation and Temporal Critique in the Italian 'It Gets Better' Project”. En Brian James Baer y Klaus Kaindl (eds.). Queering Translation-Translating the Queer. Theory, Practice, Activism. Reino Unido: Routledge, pp. 58-71.

DELABASTITA, Dirk (1996). "Introduction" en The Translator, Special Issue: Wordplay and Translation, 2(2), pp. 127-139.

HURTADO ALBIR, Amparo (2011). Traducción y traductología. Introducción a la traductología. Madrid: Ediciones Cátedra, pp. 279-289.

NORD, Christiane (1991). Text analysis in translation: theory, methodology, and didactic application of a model for translation-oriented text analysis. Ámsterdam/Nueva York: Rodopi, p. 151.

NOSSEM, Eva (2017). "Queering Lexicography: Balancing Power Relations in Dictionaries". En Brian James Baer y Klaus Kaindl (eds.). Queering TranslationTranslating the Queer. Theory, Practice, Activism. Reino Unido: Routledge, pp. $172-$ 187.

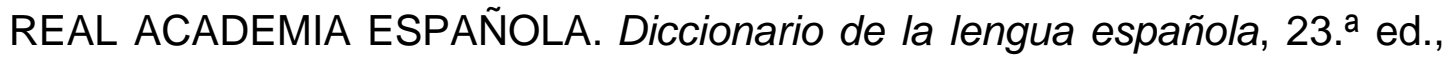
[versión 23.4 en línea]. <https://dle.rae.es/neologismo> [25/07/2021].

Revista Zona Franca- Centro de estudios interdisciplinario sobre las mujeres (CEIM)- Maestría poder y sociedad desde la problemática de género (MG), Rosario, Argentina. ISSN, 2545-6504 http://zonafranca.unr.edu.ar/index.php/ZonaFranca| Número 29 (2021). 
VON FLOTOW, Luise (1997). "Historical Background" y "Gender and the Practice of Translation". En Translation and Gender. Translating in the "Era of Feminism'. Manchester: Saint Jerome Publishing, pp. 5-34.

Revista Zona Franca- Centro de estudios interdisciplinario sobre las mujeres (CEIM)- Maestría poder y sociedad desde la problemática de género (MG), Rosario, Argentina. ISSN, 2545-6504 http://zonafranca.unr.edu.ar/index.php/ZonaFranca| Número 29 (2021). 